\title{
ON COMPLETE MONOMIAL IDEALS
}

\author{
PHILIPPE GIMENEZ, ARON SIMIS, WOLMER V. VASCONCELOS \\ AND RAFAEL H. VILLARREAL
}

\begin{abstract}
In dimension 2, we study complete monomial ideals combinatorially, their Rees algebras and develop effective means of finding their defining equations.
\end{abstract}

1. Introduction. The study of complete ideals in the polynomial ring $k[x, y]$ is a classical subject started by Zariski in [28] (see also [29, Appendix 5]) and subsequently developed by various other authors $([14,17,18,21])$.

The special case of monomial ideals is enhanced by the use of combinatorics, especially those parts related to convex hull techniques. It is somewhat surprising that, only more recently, this facet took off accordingly. Thus, in [6, 7] Quiñonez studied the normality of monomial ideals in $k[x, y]$ and established a criterion in terms of certain partial blocks and associated sequences of rational numbers.

In the present work, the overall goal is to study normal monomial ideals in $k[x, y]$ in a landscape governed by Zariski's theory of complete ideals and the structures and algorithms associated to Newton polygons. A common root between Quiñonez's approach and ours is the emphasis on the exponents of the monomials that generate the given ideal written in lexicographic order with $x>y$, thus affording a slightly different angle from the one in some of the previous classical approaches.

2010 AMS Mathematics subject classification. Primary 13B22, Secondary 13A30, $13 \mathrm{H} 10$.

Keywords and phrases. $\mathfrak{m}$-full ideal, normal ideal, Newton polytope, Hilbert function, Rees algebra, Jacobian dual, Cohen-Macaulay ring.

The first author was partially supported by the Ministerio de Economía y Competitividad (Spain), Grant number MTM2013-40775-P. The second author is partially supported by the CNPq grant 302298/2014-2 and the CAPES Senior Visiting Fellow Scholarship 5742201241/2016 at the Universidade Federal da Paraiba, Brazil.

Received by the editors on January 23, 2014, and in revised form on April 21, 2014. 
A difference between our results and Quiñonez's lies in that we state necessary or sufficient conditions for normality directly in terms of the stair sequences of the monomial exponents by means of certain inequalities. Since each of these criteria is stated by means of a finite set of numerical inequalities, it is doubtful whether one can group them together in order to obtain a full characterization of normality (this point is addressed in detail in Question 2.19).

Other points of contrast are our use of polyhedra theory (such as Pick's formula) and a strengthening of the relationship between normality and $\mathfrak{m}$-fullness-the latter a concept introduced by Rees and developed in $[\mathbf{1 5}, \mathbf{2 7}]$. Thus, the preponderance of our algebraic results is derived from the properties of the polygon defined by the points in the plane whose coordinates are the exponents of the monomials generating the ideal. This benefits from the fact that a natural starting point is the direct description of $\mathfrak{m}$-full ideals and the simplicity of their syzygies.

Unavoidably, in such a narrowly defined class of ideals coming from a slightly distinct view point, we recover some of the results of Quiñonez. In such cases, we explain the relationship between the two.

Let $\mathbf{R}=k[x, y], \mathfrak{m}=(x, y)$, and $I$ be a monomial ideal. When needed in our references to the literature, we assume $k$ is infinite. Suppose that $I$ is $\mathfrak{m}$-primary, minimally generated by $n$ elements, $\mu(I)=n$, but $I \neq \mathfrak{m}^{n-1}$. $I$ is minimally generated by $n$ monomials that are listed lexicographically, $I=\left(x^{a_{1}}, x^{a_{2}} y^{b_{n-1}}, \ldots, x^{a_{i}} y^{b_{n-i+1}}, \ldots, x^{a_{n-1}} y^{b_{2}}, y^{b_{1}}\right)$ with

$$
\begin{gathered}
a_{1}>a_{2}>\cdots>a_{n-1}>a_{n}:=0, \\
b_{1}>b_{2}>\cdots>b_{n-1}>b_{n}:=0,
\end{gathered}
$$

defining the set of points $P_{i}=\left(a_{i}, b_{n-i+1}\right), 1 \leq i \leq n$.

Our first result describes how, given a monomial ideal $I$, to find the smallest $\mathfrak{m}$-full monomial ideal $I^{\prime}$ containing it (Proposition 2.7). This works for any $\mathfrak{m}$-primary monomial ideals in $k\left[x_{1}, \ldots, x_{d}\right]$. Moreover, in the case where $d=2$, we characterize when $I$ is $\mathfrak{m}$-full (Theorem 2.9).

In a different direction, we take up the normality question, by conveying several necessary conditions or sufficient conditions for it to hold, expressed by systems of linear inequalities $Q\left(P_{1}, \ldots, P_{n}\right) \leq 0$ (Proposition 2.12, Theorem 2.13 and Proposition 2.15). 
Our most comprehensive results are given in the equations of the Rees algebras $\mathbf{R}[I t]$ of normal monomial ideals. They are put together from two facts. On one hand, the algebras $\mathbf{R}[I t]$ being normal are Cohen-Macaulay by a theorem of Lipman-Teissier ([19, Corollary 5.4]). On the other hand, the syzygies of $I$ are straightforward enough to permit getting the equations of $\mathbf{R}[I t]$ in one or two rounds of elimination. An effective application of a theorem of Morey-Ulrich ([20, Theorem 1.2]) gives the case when one round of elimination suffices (Theorem 3.3). This is an approach that has also been exploited in [3, Theorem 3.17] and [4], allied with a detailed examination of their Hilbert functions for a wider class of ideals. Here, aiming for less generality, we get to the equations as quickly and effectively as possible by introducing a second elimination round to recover them all (Theorem 3.6). Finally, we recall that, while the Rees algebras of $\mathfrak{m}$-full ideals are not always Cohen-Macaulay, they will be so if their special fiber is Cohen-Macaulay (Theorem 3.8).

\section{Criteria for $\mathfrak{m}$-fullness and normality.}

2.1. Polyhedra. Let $\mathbf{R}=k\left[x_{1}, \ldots, x_{d}\right]$ be a polynomial ring over a field $k$, with $d \geq 2$, and let $I$ be a zero-dimensional ideal of $\mathbf{R}$ minimally generated by monomials $x^{v_{1}}, \ldots, x^{v_{q}}$, where $x^{v_{j}}:=x_{1}^{v_{1, j}} \cdots x_{n}^{v_{n, j}}$, for $j=1, \ldots, q$. Consider the rational convex polyhedron $\mathcal{Q}:=\mathbb{R}_{\geq 0}^{d}+$ conv $\left(v_{1}, \ldots, v_{q}\right)$, where conv $\left(v_{1}, \ldots, v_{q}\right)$ denotes the convex hull of $v_{1}, \ldots, v_{q}$ in $\mathbb{R}^{d}$. The integral polytope $\operatorname{conv}\left(v_{1}, \ldots, v_{q}\right)$, denoted by $N(I)$, is called the Newton polytope of $I$, and $\mathcal{Q}$ is called the Newton polyhedron of $I$.

Remark 2.1. $\mathcal{Q} \cap \mathbb{Z}^{d}=\left(\mathbb{Q}_{\geq 0}^{d}+\operatorname{conv}_{\mathbb{Q}}\left(v_{1}, \ldots, v_{q}\right)\right) \cap \mathbb{Z}^{d}$. This follows using that $\mathcal{Q}$ is a rational polyhedron, i.e., the vertices of $\mathcal{Q}$ are in $\mathbb{Q}^{d}$.

As usual, we denote the floor and ceiling of a real number $r$ by $\lfloor r\rfloor$ and $\lceil r\rceil$, respectively. One can use these notions to give necessary and sufficient conditions for the normality of $I$ as well as some descriptions of the integral closures of the powers of $I[\mathbf{1}, \mathbf{8}, \mathbf{1 0}, \mathbf{1 1}]$ (see also Proposition 2.3 and Theorem 2.13 below). 
Definition 2.2. Let $A$ be the $d \times q$ integer matrix with column vectors $v_{1}, \ldots, v_{q}$. The system $x \geq 0 ; x A \geq \mathbf{1}$ of linear inequalities is said to have the integer rounding property if

$$
\max \left\{\langle y, \mathbf{1}\rangle \mid y \geq 0 ; A y \leq w ; y \in \mathbb{N}^{q}\right\}=\lfloor\max \{\langle y, \mathbf{1}\rangle \mid y \geq 0 ; A y \leq w\}\rfloor
$$

for each integer vector $w$ for which the right hand side is finite. Here, $\mathbf{1}=(1, \ldots, 1)$ and $\langle$,$\rangle denotes the usual inner product.$

Systems with the integer rounding property have been widely studied from the viewpoint of integer programming; see [22, pages 336-338], [23, pages 82-83], and the references therein.

\section{Proposition 2.3.}

(i) [8, Proposition 1.1]. $\overline{I^{m}}=\left(\left\{x^{a} \mid a \in m \mathcal{Q} \cap \mathbb{Z}^{d}\right\}\right)$ for $0 \neq m \in \mathbb{N}$.

(ii) $\left[8\right.$, Proposition 1.2]. $\bar{I}$ is generated by all $x^{a}$ with $a \in(\mathcal{Q}+$ $\left.[0,1)^{d}\right) \cap \mathbb{N}^{d}$.

(iii) [9, Corollary 2.5]. I is normal if and only if the system $x \geq$ $0 ; x A \geq \mathbf{1}$ has the integer rounding property.

Example 2.4. If $I=\left(x^{2}, y^{3}\right)$, then $N(I) \cap \mathbb{Z}^{2}=\{(2,0),(0,3)\}$ and $\bar{I}=I+\left(x y^{2}\right)$.

If $I \subset k[x, y]$, the next result will be used to give the necessary condition for $I$ to be normal (see Proposition 2.12 (ii)).

Proposition 2.5. (Pick's formula, [2, page 248]). If $\mathcal{P} \subset \mathbb{R}^{2}$ is an integral polytope of dimension 2 , then

$$
\operatorname{area}(\mathcal{P})=\left|\mathbb{Z}^{2} \cap \mathcal{P}\right|-\frac{\left|\mathbb{Z}^{2} \cap \partial \mathcal{P}\right|}{2}-1=\left|\mathbb{Z}^{2} \cap \mathcal{P}^{\mathrm{o}}\right|+\frac{\left|\mathbb{Z}^{2} \cap \partial \mathcal{P}\right|}{2}-1,
$$

where $\partial \mathcal{P}$ and $\mathcal{P}^{\circ}$ are the boundary and the interior of $\mathcal{P}$, respectively.

2.2. Full ideals. Let $\mathbf{R}=k[x, y]$ and $\mathfrak{m}=(x, y)$. An $\mathfrak{m}$-primary ideal $I$ is said to be $\mathfrak{m}$-full if $\mathfrak{m} I: a=I$ for some $a \in \mathfrak{m} \backslash \mathfrak{m}^{2}$ (see [15, subsection 14.1.5]). The element $a$ can be taken to be a linear form not dividing the content ideal $c(I)$ of $I$ (see [15, subsection 14.1.1, Proposition 14.1.7]). If $I$ is a monomial ideal, $a$ can be taken to be a 
form that is not a monomial since the content is monomial. We shall take $a=x+y$.

The fundamental characterization of $\mathfrak{m}$-full ideals of two-dimensional regular local rings is the following result due to Rees ([15, Exercise 14.1] and [27, Theorem 4]):

Theorem 2.6. Let $I$ be an $\mathfrak{m}$-primary ideal of a regular local ring $(\mathbf{R}, \mathfrak{m})$ of dimension 2. Then $I$ is $\mathfrak{m}$-full if and only if for all ideals $I \subset J, \mu(J) \leq \mu(I)$.

In analogy to the existence of the integral closure of an ideal, let us consider the question of its $\mathfrak{m}$-full closure in the sense of a unique minimal $\mathfrak{m}$-full ideal $J$ containing $I$. Since the set of $\mathfrak{m}$-full ideals containing $I$ is non-empty and satisfies the minimal chain condition there may exist, to the authors' knowledge, more than one minimal element, a situation that makes appointing one of them inappropriate as the closure. The situation is clearer if we consider only the set of monomial ideals.

Proposition 2.7. Let $I$ be an $\mathfrak{m}$-primary monomial ideal. Then its $\mathfrak{m}$-full monomial closure $I^{*}$ exists, and it is integral over $I$.

Proof. For any ideal $L$ of a polynomial ring $k\left[x_{1}, \ldots, x_{d}\right]$, we denote by $M(L)$ the ideal generated by all monomials that occur in the representation of the elements of $L$. It is clear that $M(L)$ is defined by the monomials that occur in any generating set for $L$. Note that, if $J$ is a monomial ideal and $L \subset J$, then $M(L) \subset J$.

- Consider the set of all $\mathfrak{m}$-full monomial ideals that contain the monomial ideal $I$. For each such $\mathfrak{m}$-full monomial ideal $L$, we have

$$
\mathfrak{m} I: x+y \subset \mathfrak{m} L: x+y=L .
$$

- If $\mathfrak{m} I: x+y \neq I$, that is, if $I$ is not $\mathfrak{m}$-full, note that $M(\mathfrak{m} I: x+y)$ properly contains $I$ but it is still contained in $L$. In this case, set $I_{1}=M(\mathfrak{m} I: x+y)$, and apply the previous step to it. This process defines an increasing chain of monomial ideals $I \subset I_{1} \subset I_{2} \subset \cdots$, contained in $L$ whose stable ideal $I^{*}$ is $\mathfrak{m}$-full. 
- Considering that the integral closure $\bar{I}$ of $I$ is monomial and $\mathfrak{m}$-full, we have $I^{*} \subset \bar{I}$.

Example 2.8. Let $I=\left(x^{3}, y^{5}\right)$. Then, $\mathfrak{m} I: x+y=\left(x^{3}, x^{2} y^{3}-x y^{4}, y^{5}\right)$. Thus, $I_{1}=\left(x^{3}, x^{2} y^{3}, x y^{4}, y^{5}\right)$ and $\mathfrak{m} I_{1}: x+y=I_{1}$. Thus, $I^{*}=I_{1}$.

Let us cast Theorem 2.6 for monomial ideals of $k[x, y]$ into an effective form for later usage.

Theorem 2.9. If I is minimally generated by $n$ monomials that are listed lexicographically,

$$
I=\left(x^{a_{1}}, x^{a_{2}} y^{b_{n-1}}, \ldots, x^{a_{i}} y^{b_{n-i+1}}, \ldots, x^{a_{n-1}} y^{b_{2}}, y^{b_{1}}\right),
$$

then $I$ is $\mathfrak{m}$-full if and only if there is $1 \leq k \leq n$ such that the following conditions hold:

(i) $b_{n-i}-b_{n-i+1}=1$ for $1 \leq i \leq k-1$,

(ii) $k=n$ or $k<n$ and $b_{n-k}-b_{n-k+1} \geq 2$,

(iii) $a_{i}-a_{i+1}=1$ for $k \leq i \leq n-1$.

Proof. We first show that $I$ is $\mathfrak{m}$-full if and only if order $(I)=$ $n-1$. Thus, suppose order $(I)=n-1$, i.e., there is an element $x^{k} y^{n-1-k} \in I$. Note that $I \subset J$ implies order $(J) \leq \operatorname{order}(I)$. Since $\mu(J) \leq \operatorname{order},(J)+1 \leq n, I$ satisfies Theorem 2.6.

Conversely, if $I$ is $\mathfrak{m}$-full, order $(I) \leq n-1$, as otherwise $I \subset(x, y)^{n}$, which has $n+1$ minimal generators, which would violate Theorem 2.6.

Now, granted order $(I)=n-1$, suppose an occurrence of a monomial of degree $n-1$ is $x^{k} y^{n-1-k}$. This means that there are at most $n-1-k$ elements prior to $x^{k} y^{n-1-k}$ and at most $k$ elements after. This gives

$$
I=\left(x^{a_{1}}, x^{a_{2}} y, \ldots, x^{a_{k-1}} y^{n-2-k}, x^{k} y^{n-1-k}, x^{k-1} y^{b_{n-k}}, \ldots, x y^{b_{2}}, y^{b_{1}}\right) .
$$

By choosing $k$ as small as possible we achieve all three conditions.

Conversely, it is clear that the set of the three stated conditions implies order $(I)=n-1$.

Corollary 2.10. Let I be an ideal minimally generated by $n$ monomials that are listed lexicographically, $I=\left(x^{a_{1}}, x^{a_{2}} y^{b_{n-1}}, \ldots, x^{a_{i}} y^{b_{n-i+1}}, \ldots\right.$, $\left.x^{a_{n-1}} y^{b_{2}}, y^{b_{1}}\right)$. Suppose that I is normal. 
(i) For every $i$, either $a_{i}-a_{i+1}=1$ or $b_{n-i}-b_{n-i+1}=1$.

(ii) If $b_{n-i}-b_{n-i+1}>1$ for some $i$, then $a_{i}-a_{i+1}=1$ and $a_{i+1}-a_{i+2}=1$.

Proof. Both claims follow readily from Theorem 2.9 because complete monomial ideals of $k[x, y]$ are $\mathfrak{m}$-full [15, Theorem 14.1.8].

This result has already been observed in [7, page 369]. In the same work, the following terminology was introduced for zero-dimensional monomial ideals $I \subset k[x, y]$, whose generators are ordered as above: $I$ is called $x$-tight (respectively, $y$-tight) if $a_{i}-a_{i+1}=1$ for all $i$ (respectively, $b_{i}-b_{i+1}=1$ for all $i$ ).

Putting together the previous result and these notions, we have:

Corollary 2.11. If I as above is $\mathfrak{m}$-full, then it is the product of an $x$-tight ideal and a $y$-tight ideal.

Proof. It follows from Theorem 2.9 and [7, Proposition 2.2].

2.3. Normality criteria. We now proceed to establish separate necessary or sufficient conditions for normality in terms of the associated monomial exponents. First we state the necessary conditions:

Proposition 2.12. Let $I$ be an ideal minimally generated by $n$ monomials that are listed lexicographically, $I=\left(x^{a_{1}}, x^{a_{2}} y^{b_{n-1}}, \ldots, x^{a_{i}} y^{b_{n-i+1}}\right.$, $\left.\ldots, x^{a_{n-1}} y^{b_{2}}, y^{b_{1}}\right)$, and let $P_{i}=\left(a_{i}, b_{n-i+1}\right), P_{i+1}=\left(a_{i+1}, b_{n-i}\right)$ and $P_{i+2}=\left(a_{i+2}, b_{n-i-1}\right)$ be three consecutive points corresponding to the exponents of the defining monomials of $I$. The following hold:

(i) If $I=\bar{I}$ and $b_{n-i-1}-b_{n-i}=1, b_{n-i}-b_{n-i+1}=1$, then $a_{i+1} \leq\left\lceil\left(a_{i}+a_{i+2}\right) / 2\right\rceil$.

(ii) If $I=\bar{I}$ and $a_{i}-a_{i+1}=1, a_{i+1}-a_{i+2}=1$, then $b_{n-i} \leq$ $\left\lceil\left(b_{n-i-1}+b_{n-i+1}\right) / 2\right\rceil$.

Proof. (i) First we assume that $P_{i+1} \in \operatorname{conv}\left(P_{i}, P_{i+2}\right)$. Then, we can write $P_{i+1}=\lambda_{1} P_{i}+\lambda_{2} P_{i+2}$, where $\lambda_{i}>0, i=1,2$ and $\lambda_{1}+\lambda_{2}=1$. It is not hard to see that $\lambda_{i}=1 / 2$ for $i=1,2$. Thus, one has

$$
a_{i+1}=\frac{a_{i}+a_{i+2}}{2}=\left\lceil\frac{a_{i}+a_{i+2}}{2}\right\rceil \text {. }
$$


We may now assume that $P_{i+1} \notin \operatorname{conv}\left(P_{i}, P_{i+2}\right)$. We proceed by contradiction assuming that

$$
a_{i+1}>\left\lceil\frac{a_{i}+a_{i+2}}{2}\right\rceil, \quad \text { that is, } \quad a_{i+1}-\frac{a_{i}+a_{i+2}}{2} \geq 1 .
$$

Consider the convex polytope $\mathcal{P}$ whose vertices are $P_{i}, P_{i+1}, P_{i+2}$. We claim that $\partial \mathcal{P} \cap \mathbb{Z}^{2}=\left\{P_{i}, P_{i+1}, P_{i+2}\right\}$. Clearly, conv $\left(P_{i}, P_{i+1}\right)^{\circ} \cap \mathbb{Z}^{2}=\emptyset$ and $\operatorname{conv}\left(P_{i+1}, P_{i+2}\right)^{\circ} \cap \mathbb{Z}^{2}=\emptyset$, because $b_{n-i-1}-b_{n-i}=1$ and $b_{n-i}-b_{n-i+1}=1$. We claim that also $\operatorname{conv}\left(P_{i}, P_{i+2}\right)^{\circ} \cap \mathbb{Z}^{2}=\emptyset$. Indeed, if this set is non-empty, pick an integral point $\left(c_{1}, c_{2}\right)$ in $\operatorname{conv}\left(P_{i}, P_{i+2}\right)^{\mathrm{o}}$. By Proposition 2.3, the monomial $x^{c_{1}} y^{c_{2}}$ is in $\bar{I}=I$. Then, we can write

$$
\begin{aligned}
& c_{1}=t a_{i+2}+(1-t) a_{i}=\epsilon_{1}+a_{j}, \\
& c_{2}=t b_{n-i-1}+(1-t) b_{n-i+1}=\epsilon_{2}+b_{n-j+1},
\end{aligned}
$$

for some $j$, where $\epsilon_{1}$ and $\epsilon_{2}$ are in $\mathbb{N}$ and $0<t<1$. From equation (2.1), we get $a_{i}>c_{1}=\epsilon_{1}+a_{j}$. Thus, $i<j$. From equation (2.2), we get

$$
c_{2}=t\left(b_{n-i-1}-b_{n-i+1}\right)+b_{n-i+1}=2 t+b_{n-i+1}=\epsilon_{2}+b_{n-j+1} .
$$

Hence, $2+b_{n-i+1}>\epsilon_{2}+b_{n-j+1}$. If $\epsilon_{2} \geq 1$, then $b_{n-i+1}-b_{n-j+1} \geq 0$, and consequently, $i \geq j$, a contradiction. Hence, $\epsilon_{2}=0, j=i+1$ and $t=1 / 2$. Therefore, from equation (2.1), we obtain

$$
\epsilon_{1}=\frac{a_{i}+a_{i+2}}{2}-a_{i+1} \geq 0,
$$

a contradiction. This completes the proof of the claim. As a consequence, using Pick's formula (Proposition 2.5), one has

$$
\operatorname{area}(\mathcal{P})=\left|\mathcal{P}^{0} \cap \mathbb{Z}^{2}\right|+\frac{1}{2} .
$$

The equation of the line passing through $P_{i}$ and $P_{i+2}$ is

$$
\begin{aligned}
& x_{1}\left(b_{n-i-1}-b_{n-i+1}\right)+x_{2}\left(a_{i}-a_{i+2}\right) \\
& \quad=a_{i}\left(b_{n-i-1}-b_{n-i+1}\right)+\left(a_{i}-a_{i+2}\right) b_{n-i+1} .
\end{aligned}
$$

Since

$$
a_{i+1}-\frac{a_{i}+a_{i+2}}{2} \geq 1
$$


the point $P_{i+1}$ lies above this line. It follows readily that the area of $\mathcal{P}$ is given by

$$
\operatorname{area}(\mathcal{P})=a_{i+1}-\frac{a_{i}+a_{i+2}}{2} \geq 1
$$

Hence, by equation $(2.3), \mathcal{P}^{0} \cap \mathbb{Z}^{2} \neq \emptyset$. Pick an integral point $\left(c_{1}, c_{2}\right)$ in $\mathcal{P}^{0}$. By Proposition 2.3, the monomial $x^{c_{1}} y^{c_{2}}$ is in $\bar{I}=I$. Then we can write

$$
\begin{aligned}
& c_{1}=\lambda_{1} a_{i}+\lambda_{2} a_{i+1}+\lambda_{3} a_{i+2}=\epsilon_{1}+a_{j}, \\
& c_{2}=\lambda_{1} b_{n-i+1}+\lambda_{2} b_{n-i}+\lambda_{3} b_{n-i-1}=\epsilon_{2}+b_{n-j+1},
\end{aligned}
$$

for some $j$, where $\epsilon_{1}$ and $\epsilon_{2}$ are in $[0,1), \lambda_{i}>0$ for $i=1,2,3$, and $\lambda_{1}+\lambda_{2}+\lambda_{3}=1$. From equations (2.4) and (2.5), we get $a_{i}>c_{1}=\epsilon_{1}+a_{j}$ and $b_{n-i-1}>c_{2}=\epsilon_{2}+b_{n-j+1}$. Thus, $i<j$ and $-2<i-j$, i.e., $j=i+1$. Therefore, we can rewrite equation (2.5) as

$$
\begin{aligned}
c_{2} & =\lambda_{1}\left(b_{n-i}-1\right)+\lambda_{2} b_{n-i}+\lambda_{3}\left(b_{n-i}+1\right) \\
& =b_{n-i}-\lambda_{1}+\lambda_{3}=\epsilon_{2}+b_{n-i} .
\end{aligned}
$$

As a consequence, $-\lambda_{1}+\lambda_{3}=\epsilon_{2} \geq 0$. Hence, $\epsilon_{2}$ must be zero because $\epsilon_{2}<\lambda_{3}<1$. Then, from equation (2.4), we get

$$
\begin{aligned}
c_{1} & =\lambda_{1}\left(a_{i}+a_{i+2}\right)+\lambda_{2} a_{i+1} \\
& =\lambda_{1}\left(a_{i}+a_{i+2}\right)+\left(1-2 \lambda_{1}\right) a_{i+1}=\epsilon_{1}+a_{i+1} .
\end{aligned}
$$

Thus, $\lambda_{1}\left(a_{i}+a_{i+2}-2 a_{i+1}\right)=\epsilon_{1} \geq 0$, and hence, $a_{i}+a_{i+2}-2 a_{i+1} \geq 0$, a contradiction.

(ii) Notice that the ideal obtained from $I$ by permuting $x$ and $y$ is also normal. Thus, this part follows from (i).

Putting together Corollary 2.10 and Proposition 2.12 we obtain the following:

Theorem 2.13. Let I be minimally generated by $n$ monomials that are listed lexicographically, $I=\left(x^{a_{1}}, x^{a_{2}} y^{b_{n-1}}, \ldots, x^{a_{i}} y^{b_{n-i+1}}, \ldots, x^{a_{n-1}} y^{b_{2}}\right.$, $\left.y^{b_{1}}\right)$. If $I$ is normal, then there exists $k, 1 \leq k \leq n$, such that:

(i) $a_{n-1}=1, a_{n-2}=2, \ldots, a_{k}=n-k$,

(ii) $b_{n-1}=1, b_{n-2}=2, \ldots, b_{n-k+1}=k-1$,

(iii) $b_{2} \leq\left\lceil\left(b_{1}+b_{3}\right) / 2\right\rceil, b_{3} \leq\left\lceil\left(b_{2}+b_{4}\right) / 2\right\rceil, \ldots, b_{n-k} \leq\left\lceil\left(b_{n-k-1}\right.\right.$ $\left.\left.+b_{n-k+1}\right) / 2\right\rceil$, 
(iv) $a_{2} \leq\left\lceil\left(a_{1}+a_{3}\right) / 2\right\rceil, a_{3} \leq\left\lceil\left(a_{2}+a_{4}\right) / 2\right\rceil, \ldots, a_{k-1} \leq\left\lceil\left(a_{k-2}+\right.\right.$ $\left.\left.a_{k}\right) / 2\right\rceil$.

Proof. There is a $1 \leq k \leq n$ such that $b_{n-1}=1, b_{n-2}=$ $2, \ldots, b_{n-k+1}=k-1$ and $b_{n-k}-b_{n-k+1} \geq 2$. Then, using Corollary 2.10 (ii), it is seen that $a_{i}-a_{i+1}=1$ for $i \geq k$. Hence, (i) and (ii) hold. Parts (iii) and (iv) follow from Proposition 2.12.

Example 2.14. The ideal $I=\left(x^{3}, x^{2} y^{8}, x y^{15}, y^{21}\right)$ is not normal (but it is $\mathfrak{m}$-full) and satisfies the conditions of Theorem 2.13. The integral closure of $I$ is $\bar{I}=\left(x^{3}, x^{2} y^{7}, x y^{14}, y^{21}\right)$.

We next state sufficient conditions of a similar nature for normality.

Proposition 2.15. Let $I \subset K[x, y]$ be an ideal minimally generated by $n$ monomials that are listed lexicographically,

$$
I=\left(x^{a_{1}}, x^{a_{2}} y^{b_{n-1}}, \ldots, x^{a_{i}} y^{b_{n-i+1}}, \ldots, x^{a_{n-1}} y^{b_{2}}, y^{b_{1}}\right) .
$$

If $a_{i}-a_{i+1}=1$ for $i=1, \ldots, n-1$, and $2 b_{n-i} \leq b_{n-i-1}+b_{n-i+1}$ for all $i$, then $I$ is normal.

Proof. Notice that $a_{i}=n-i$ for $i=1, \ldots, n$. Let $x^{c_{1}} y^{c_{2}}$ be a minimal monomial generator of $\bar{I}$. By Proposition 2.3 (ii), we can write

$$
\begin{aligned}
& c_{1}=\lambda_{1}(n-1)+\cdots+\lambda_{i}(n-i)+\cdots+\lambda_{n-1}(1)+\epsilon_{1}, \\
& c_{2}=\lambda_{2} b_{n-1}+\cdots+\lambda_{i} b_{n-i+1}+\cdots+\lambda_{n-1} b_{2}+\lambda_{n} b_{1}+\epsilon_{2},
\end{aligned}
$$

where $\epsilon_{1}$ and $\epsilon_{2}$ are in $[0,1), \lambda_{i} \geq 0$ for all $i$, and $\sum_{i=1}^{n} \lambda_{i}=1$. Hence, $0 \leq c_{1}<n$. As $c_{1}$ is an integer, one has $0 \leq c_{1} \leq n-1$. Thus, $c_{1}=a_{i}=n-i$ for some $1 \leq i \leq n$. To show that $x^{c_{1}} y^{c_{2}}$ is in $I$ it suffices to show that $x^{c_{1}} y^{c_{2}}$ is a multiple of $x^{a_{i}} y^{b_{n-i+1}}$. The proof reduces to showing that $c_{2} \geq b_{n-i+1}$. Thus, by equation (2.7), we need only show the following inequality

$$
\begin{aligned}
\lambda_{2} b_{n-1}+ & \cdots+\lambda_{i-1} b_{n-i+2}+\lambda_{i+1} b_{n-i}+\cdots \\
& +\lambda_{n-1} b_{2}+\lambda_{n} b_{1} \geq\left(1-\lambda_{i}\right) b_{n-i+1} .
\end{aligned}
$$


Using $1-\lambda_{i}=\sum_{j \neq i} \lambda_{j}$, it follows that this inequality is equivalent to $(2.9)$

$$
\begin{gathered}
\lambda_{i+1}\left(b_{n-i}-b_{n-i+1}\right)+\lambda_{i+2}\left(b_{n-i-1}-b_{n-i+1}\right)+\cdots+\lambda_{n}\left(b_{1}-b_{n-i+1}\right) \\
\geq \lambda_{1} b_{n-i+1}+\lambda_{2}\left(b_{n-i+1}-b_{n-1}\right)+\cdots+\lambda_{i-1}\left(b_{n-i+1}-b_{n-i+2}\right) .
\end{gathered}
$$

From equation (2.6) and using the equality $n-i=(n-i) \sum_{j=1}^{n} \lambda_{j}$, one has

$$
\begin{aligned}
\lambda_{i+1}(1) \geq & \lambda_{1}(i-1)+\cdots+\lambda_{i-1}(1) \\
& -\left[\lambda_{i+2}(2)+\cdots+\lambda_{n-1}(n-i-1)+\lambda_{n}(n-i)\right] .
\end{aligned}
$$

Hence, to show equation (2.9), it suffices to prove the following inequality:

$$
\begin{aligned}
\lambda_{1}\left[(i-1)\left(b_{n-i}-b_{n-i+1}\right)-b_{n-i+1}\right]+\cdots \\
\quad+\lambda_{i-1}\left[\left(b_{n-i}-b_{n-i+1}\right)-\left(b_{n-i+1}-b_{n-i+2}\right)\right] \\
\quad+\lambda_{i+2}\left[\left(b_{n-i-1}-b_{n-i+1}\right)-2\right]+\cdots \\
\quad+\lambda_{n}\left[\left(b_{1}-b_{n-i+1}\right)-(n-i)\right] \geq 0 .
\end{aligned}
$$

To complete the proof, notice that this inequality holds because all coefficients of $\lambda_{1}, \ldots, \lambda_{n}$ are non-negative.

Example 2.16. Let $I$ be the ideal of $\mathbb{Q}[x, y]$ generated by $x^{2}, x y^{2}, y^{3}$. This ideal is normal, and satisfies $a_{i}-a_{i+1}=1$ for $i=1,2$, but $2 b_{2} \not \leq b_{1}+b_{3}$, where $b_{1}=3, b_{2}=2$ and $b_{3}=0$.

Corollary 2.17. Let I be minimally generated by $n$ monomials that are listed lexicographically, $I=\left(x^{a_{1}}, x^{a_{2}} y^{b_{n-1}}, \ldots, x^{a_{i}} y^{b_{n-i+1}}, \ldots, x^{a_{n-1}} y^{b_{2}}\right.$, $\left.y^{b_{1}}\right)$. Assume that $I$ is $\mathfrak{m}$-full, and let $k$ be the integer obtained in Theorem $2.9,1 \leq k \leq n$. If

(i) $2 b_{2} \leq b_{1}+b_{3}, 2 b_{3} \leq b_{2}+b_{4}, \ldots, 2 b_{n-k} \leq b_{n-k-1}+b_{n-k+1}$,

(ii) $2 a_{2} \leq a_{1}+a_{3}, 2 a_{3} \leq a_{2}+a_{4}, \ldots, 2 a_{k-1} \leq a_{k-2}+a_{k}$,

then $I$ is normal.

Proof. As observed in Corollary 2.11, an $\mathfrak{m}$-full ideal $I$ is the product of an $x$-tight ideal $X$ and a $y$-tight ideal $Y$. Moreover, by [7, Proposition 2.6], the product of an $x$-tight ideal and a $y$-tight ideal is integrally closed if and only if both ideals are integrally closed. One can apply 
Proposition 2.15 to $X$, and the similar result holds for $y$-tight ideals to $Y$ to get the required result.

Remark 2.18. For an $\mathfrak{m}$-full ideal, normality is a condition in between the set of conditions (iii)-(iv) in Theorem 2.13, and the set of conditions (i)-(ii) in Corollary 2.17.

\section{Related questions.}

Question 2.19 (Finiteness question). Each of the necessary and sufficient conditions of normality above is cast in the form of a system $Q$ of linear inequalities on the coordinates of the points $P_{i}$. It is not likely that a full set of conditions can be expressed by a finite set $Q_{1}, \ldots, Q_{m}$ of inequalities. More precisely, for each type of such inequality $Q$, denote by $M(Q)$ the set of all monomial ideals that satisfies $Q$. For instance, for the normal ideals I lying in the variety $M\left(Q_{i}\right)$, then for all pairs of integers $a, b \geq 1$, the ideal $\left(x^{a}, y\right)\left(x, y^{b}\right) I$ is also normal, by Zariski's theorem, so it must belong to one of the other varieties $M\left(Q_{j}\right)$.

Question 2.20 (Realization question). Let I be an $\mathfrak{m}$-full ideal minimally generated by $n$ elements and $\bar{I}$ its integral closure. Since $\bar{I}$ is also minimally generated by $n$ elements, there is at least one map $\varphi$ between the set of points $\left\{P_{1}, \ldots, P_{n}\right\}$ of $I$ and $\left\{P_{1}^{\prime}, \ldots, P_{n}^{\prime}\right\}$ of $\bar{I}$ given by $P_{i}=P_{j}^{\prime}+R_{i j}$, for each $i$ and some $j$. Note that $R_{1 j}=R_{n j}=(0,0)$. We ask what is the nature of such maps? Is there more than one such mapping? A positive answer would help in predicting the integral closure of a monomial ideal by first determining its $\mathfrak{m}$-full closure.

3. Rees algebras. Let $I$ be a monomial ideal of $\mathbf{R}=k[x, y]$. We now study the Rees algebras $\mathbf{R}[I t]$ emphasizing when they are CohenMacaulay and obtaining their defining equations.

3.1. Syzygies. We have the following facts about their syzygies.

(Matrix of syzygies). Let $I$ be an ideal minimally generated by $n$ monomials that are listed lexicographically,

$$
I=\left(x^{a_{1}}, x^{a_{2}} y^{b_{n-1}}, \ldots, x^{a_{i}} y^{b_{n-i+1}}, \ldots, x^{a_{n-1}} y^{b_{2}}, y^{b_{1}}\right) .
$$


Among the Taylor syzygies, a subset of $n-1$ "consecutive" ones minimally generate, giving rise to the $n \times(n-1)$ syzygy matrix:

$$
\varphi=\left[\begin{array}{rrrrr}
y^{b_{n-1}} & 0 & \cdots & 0 & 0 \\
-x^{a_{1}-a_{2}} & y^{b_{n-2}-b_{n-1}} & \cdots & 0 & 0 \\
0 & -x^{a_{2}-a_{3}} & \cdots & 0 & 0 \\
\vdots & \vdots & \cdots & \vdots & \vdots \\
0 & 0 & \cdots & y^{b_{2}-b_{3}} & 0 \\
0 & 0 & \cdots & -x^{a_{n-2}-a_{n-1}} & y^{b_{1}-b_{2}} \\
0 & 0 & \cdots & 0 & -x^{a_{n-1}}
\end{array}\right] .
$$

Note that $\varphi$ is monomial (this is not typical of monomial ideals in higher dimension, it is an issue of which Cohen-Macaulay monomial ideals of codimension 2 have a minimal presentation with monomial entries). In particular, we have:

Proposition 3.1. Let $I$ be a codimension 2 monomial ideal of $\mathbf{R}=$ $k[x, y]$. The content ideal of the syzygies of $I$ is $I_{1}(\varphi)=\left(x^{r}, y^{s}\right)$.

A similar assertion holds for an $\mathfrak{m}$-primary $\mathfrak{m}$-full ideal of a twodimensional regular local $\operatorname{ring}(\mathbf{R}, \mathfrak{m})$ (of infinite residue field): $I_{1}(\varphi)=$ $(x, f), x \in \mathfrak{m} \backslash \mathfrak{m}^{2}$.

3.2. Equations of the Rees algebra. Let $I$ be an ideal of $\mathbf{R}$ minimally generated by $n$ monomials. Let $\mathbf{B}=\mathbf{R}\left[\mathbf{T}_{1}, \ldots, \mathbf{T}_{n}\right] \mapsto \mathbf{R}[I t]$ be an $R$-algebra presentation of the Rees algebra of $I$, and set $Q$ to be the kernel. $Q$ is a graded prime ideal in the standard $R$-grading of $\mathbf{B}$, $Q=Q_{1}+Q_{2}+\cdots$. With the syzygies defining $Q_{1}$, we focus on $Q_{2}$.

- (Elimination). Write the set $Q_{1}$ of syzygies of $I$ as

$$
Q_{1}=\left[\mathbf{T}_{1}, \ldots, \mathbf{T}_{n}\right] \cdot \varphi=\mathbf{T} \cdot \varphi,
$$

which we rewrite as

$$
\mathbf{T} \cdot \varphi=I_{1}(\varphi) \cdot \mathbf{B}(\varphi),
$$

$(\mathbf{B}(\varphi)$ is called the Jacobian dual of $\varphi)$ where $I_{1}(\varphi)$ is represented as $\left[x^{r}, y^{s}\right]$. By elimination,

$$
I_{2}(\mathbf{B}(\varphi)) \subset Q_{2} \subset Q .
$$


- (Expected equations). $I$ is said to have the expected equations if $Q=\left(Q_{1}, I_{2}(\mathbf{B}(\varphi))\right)$. Our setting is now ready for several applications of [20]. See also [3, Theorem 3.17], where a similar development takes place.

We will make use of the following criterium of Cohen-Macaulayness of Rees algebras.

Proposition 3.2 (Cohen-Macaulay test). Let $(\mathbf{R}, \mathfrak{m})$ be a CohenMacaulay local ring of dimension 2 and infinite residue field. If I is an $\mathfrak{m}$-primary ideal, then $\mathbf{R}[I t]$ is Cohen-Macaulay if and only if the reduction number of $I$ is at most 1 .

Proof. The forward assertion is a consequence of the Goto-Shimoda theorem ([12]) for rings of dimension 2. For the converse, if $J$ is a minimal reduction and $I^{2}=J I, I \mathbf{R}[I t]=I \mathbf{R}[J t]$, from which it follows that $I \mathbf{R}[I t]$ is a maximal Cohen-Macaulay module over $\mathbf{R}[I t]$. The Cohen-Macaulayness of $\mathbf{R}[I t]$ follows from this (see [26, page 102]).

Theorem 3.3. The Rees algebra of a complete ideal I of $\mathbf{R}$ is always Cohen-Macaulay. In particular, I has reduction number $\leq 1$. I has the expected equations if and only if

$$
I_{n-2}(\varphi)=I_{1}(\varphi)^{n-2} .
$$

The Cohen-Macaulayness is the result of Lipman-Teissier ([19, Corollary 5.4]). The last assertion follows from [20, Theorem 1.2] and the observations above on the syzygies of $I$.

Example 3.4. Suppose $I=\left(x, y^{b_{1}}\right) \cdots\left(x, y^{b_{n-1}}\right), b_{1} \leq \cdots \leq b_{n-1}$, $n \geq 4$. Consider its matrix $\varphi$ of syzygies. Inspection gives: $I_{1}(\varphi)=$ $\left(x, y^{b_{1}}\right)$, while the required equality

$$
I_{n-2}(\varphi)=I_{1}(\varphi)^{n-2},
$$

that is,

$$
\left(x^{n-2}, x^{n-3} y^{b_{1}}, x^{n-4} y^{b_{1}+b_{2}}, \ldots, y^{b_{1}+b_{2}+\cdots+b_{n-1}}\right)=\left(x, y^{b_{1}}\right)^{n-2},
$$

means 


$$
b_{1}=b_{2}=\cdots=b_{n-1}
$$

and therefore, $I=\left(x, y^{b_{1}}\right)^{n-1}$.

These observations mean that, at least among standard ideals, those with the expected equations are rare. If $I$ is normal but does not have the expected equations, where are the missing equations? A guess (to be proved below) is that they are quadratic, missing from $I_{2}(\mathbf{B}(\varphi))$. Note that, if $I$ has the expected equations,

$$
Q=I_{1}(\varphi) \mathbf{B}(\varphi): I_{1}(\varphi)
$$

Since the right-hand side is always contained in $Q$, we now discuss the case of equality. If $I$ has the expected equations, $K=\mathbf{T} \cdot \varphi+I_{2}(\mathbf{B}(\varphi))$ is a prime ideal of $\mathbf{R}[\mathbf{T}]$ of height $n-1$. We can rewrite $(K,(x, y))$ (an ideal of height $n$ ) as

$$
(K+(x, y))=(L,(x, y)),
$$

where $L$ is the ideal of $k[\mathbf{T}]$ of the maximal minors of the $2 \times(n-1)$ matrix $\mathbf{B}_{0}(\varphi)$ obtained from $\mathbf{B}(\varphi)$ by reduction $\bmod (x, y)$. By the Eagon-Northcott formula,

$$
\text { height } L \leq(n-1)-2+1=n-2 \text {. }
$$

The equality height $L=n-2$ now follows from (3.1). Thus, $L$ is Cohen-Macaulay. We note that, with this, we have that the regularity of $k[\mathbf{T}] / L$ is 1 , since $\mathbf{B}_{0}(\varphi)$ is a matrix with linear entries.

Theorem 3.5. Let I be a monomial ideal such that $\mathbf{R}[I t]$ is CohenMacaulay. Let $\varphi$ be the matrix of syzygies of $I$ and $\mathbf{B}_{0}(\varphi)$ the matrix of linear forms of $k[\mathbf{T}]$ defined above. The following conditions are equivalent:

(i) I has the expected equations;

(ii) height $I_{2}\left(\mathbf{B}_{0}(\varphi)\right)=n-2$.

Proof. It suffices to show that (ii) implies (i). We will prove this by showing that $K=\left(\mathbf{T} \cdot \varphi, I_{2}(\mathbf{B}(\varphi))\right.$ is a prime ideal. Since height $(K,(x, y))=n$, height $K \geq n-2$. Let $P$ be a minimal prime of $K$ of height $n-2,(x, y) \not \subset P$. Let $z \in(x, y) \backslash P$. Then the localization 
$P_{z}$ is a minimal prime of $K_{z}=\left(I_{n-1}(\varphi) \cdot \mathbf{T}\right)_{z}$. But this is the defining ideal of $\mathbf{R}_{z}[t]$, so it has height $n-1$.

This shows that $K$ has height $n-1 . K$ is a specialization of a generic residual intersection of a complete intersection, so it is Cohen-Macaulay ([16, Theorem 5.9]).

To prove $Q=K$, it suffices to show that $K$ is prime (recall that $Q$ is a prime of height $n-1)$. As above, we can pick $z \in(x, y)$ but avoiding every associated prime of $K$. But, as we saw, $K_{z}$ is a prime ideal of height $n-1$. This is enough to show that $K$ is prime.

3.3. Full set of quadratic equations. We shall describe where the quadratic relations of the Rees algebras $\mathbf{R}[I t]$ are located. In general, from a presentation

$$
0 \longrightarrow Q \longrightarrow \mathbf{B}=\mathbf{R}\left[\mathbf{T}_{1}, \ldots, \mathbf{T}_{n}\right] \longrightarrow \mathbf{R}[I t] \longrightarrow 0,
$$

B/ $\left./ Q_{1}\right)$ defines the symmetric algebra $\operatorname{Sym}(I)$ of $I$. We put

$$
0 \longrightarrow \mathcal{A}=A_{2}+A_{3}+\cdots \longrightarrow \operatorname{Sym}(I) \longrightarrow \mathbf{R}[I t] \longrightarrow 0 .
$$

Here, $A_{2}$ represents the effective quadratic relations of the Rees algebra $\mathbf{R}[I t]$, and we represent it as

$$
0 \longrightarrow \delta(I) \longrightarrow S_{2}(I) \longrightarrow I^{2} \longrightarrow 0 .
$$

For a discussion of $\delta(I)$, see [24]. One of its properties gives $\delta(I)$ in the exact sequence

$$
0 \longrightarrow \delta(I) \longrightarrow \mathrm{H}_{1}(I) \longrightarrow(\mathbf{R} / I)^{n} \longrightarrow I / I^{2} \longrightarrow 0,
$$

where $\mathrm{H}_{1}(I)$ is the first Koszul homology module on a set of $n$ generators of $I$. This says that $\delta(I)$ are the homology classes of the syzygies of $I$ with coefficients in $I$.

Theorem 3.6. Let $(\mathbf{R}, \mathfrak{m})$ be a two-dimensional regular local ring and $I$ an $\mathfrak{m}$-primary ideal. If the Rees algebra $\mathbf{R}[I t]$ is Cohen-Macaulay, then

$$
Q=\left(Q_{1}, Q_{2}\right)=\left(Q_{1}\right): I=(\mathbf{T} \cdot \varphi): I .
$$

Proof. Since $\mathbf{R}$ is Cohen-Macaulay, the reduction number $r(I)$ of $I$ satisfies $r(I)<\operatorname{dim} \mathbf{R}=2$. We now apply [25, Theorem 1.2]: $\mathbf{R}[I t]$ is 
defined by linear and quadratic equations, $Q=\left(Q_{1}, Q_{2}\right)$ and

$$
\operatorname{ann}(\delta(I)) \cdot Q_{2} \subset Q_{1} \mathbf{B}_{1} .
$$

Of course, any nonzero ideal contained in $\operatorname{ann}(\delta(I))$ serves the purpose, in particular ann $\left(\mathrm{H}_{1}(I)\right) \supset I$ (actually there is equality). This gives the assertion.

Note that this does not require that $I_{1}(\varphi)$ be a complete intersection.

Example 3.7. Let

$$
I=(x, y)\left(x, y^{3}\right)\left(x, y^{6}\right)=\left(x^{3}, x^{2} y, x y^{4}, y^{10}\right)
$$

$I$ is normal, and its matrix of syzygies is

$$
\varphi=\left[\begin{array}{ccc}
y & 0 & 0 \\
-x & y^{3} & 0 \\
0 & -x & y^{6} \\
0 & 0 & -x
\end{array}\right]
$$

Note that $I_{1}(\varphi)=(x, y)$, but $I_{2}(\varphi)=\left(x^{2}, x y, y^{4}\right) \neq I_{1}(\varphi)^{2}$, so it does not have the expected equations. We have:

$$
I_{2}(\mathbf{B}(\varphi))=I_{2}\left(\left[\begin{array}{ccc}
-\mathbf{T}_{2} & -\mathbf{T}_{3} & -\mathbf{T}_{4} \\
\mathbf{T}_{1} & y^{2} \mathbf{T}_{2} & y^{5} \mathbf{T}_{3}
\end{array}\right]\right),
$$

which gives only two minimal generators for $Q_{2}$. An appeal to Macaulay2 ([13]) gives the extra generator:

$$
\begin{aligned}
Q=\left(Q_{1}, Q_{2}\right) & =\mathbf{T} \cdot \varphi: I \\
& =\left(\mathbf{T} \cdot \varphi, I_{2}(\mathbf{B}(\varphi)), \mathbf{T}_{2} \mathbf{T}_{4}-y^{3} \mathbf{T}_{3}^{2}\right) .
\end{aligned}
$$

An interesting question would be about the arithmetical and homological properties of the Rees algebras of $\mathfrak{m}$-full ideals. Even for monomial ideals, these often fail to be Cohen-Macaulay, as the following example shows:

$$
I=\left(x^{11}, x^{8} y, x^{6} y^{2}, x^{5} y^{3}, x y^{4}, y^{10}\right),
$$

an $\mathfrak{m}$-full ideal. To show that $\mathbf{R}[I t]$ is not Cohen-Macaulay, by invoking Macaulay2, it is enough to verify that the special fiber $\mathcal{F}(I)$ of $I$ is not 
Cohen-Macaulay, according to the following criterion inspired by $[\mathbf{5}$, Corollary 2.11]:

Theorem 3.8. Let $I$ be an $\mathfrak{m}$-primary $\mathfrak{m}$-full ideal. If the special fiber $F=\mathcal{F}(I)$ is Cohen-Macaulay, then $\mathbf{R}[I t]$ is also Cohen-Macaulay.

Proof. Suppose $\mu(I)=n$, and let us determine the Hilbert function of $F$. For every $j \geq 0$,

$$
\mu\left(F_{j}\right)=j(n-1)+1
$$

since $I^{j}$ is $\mathfrak{m}$-full and contains an element of order $j(n-1)$. It follows that the Hilbert series of $F$ is:

$$
H_{F}(\mathbf{t})=\frac{1+(n-2) \mathbf{t}}{(1-\mathbf{t})^{2}} .
$$

This says that if $F$ is Cohen-Macaulay, as a module over a Noetherian normalization $\mathbf{A}=k[u, v]$, it is $\mathbf{A}$-free, with one generator of degree 0 and $n-2$ generators of degrees

$$
1 \leq d_{1} \leq d_{2} \leq \cdots \leq d_{n-2}
$$

The Hilbert function forces $d_{1}=\cdots=d_{n-2}=1$. Therefore, $I$ has reduction number at most one.

The same assertion holds for two-dimensional regular local rings of infinite residue field.

\section{REFERENCES}

1. J.P. Brennan, L.A. Dupont and R.H. Villarreal, Duality, a-invariants and canonical modules of rings arising from linear optimization problems, Bull. Math. Soc. Sci. Math. Roum. 51 (2008), 279-305.

2. W. Bruns and J. Gubeladze, Polytopes, rings, and K-theory, Springer Mono. Math., Springer, Dordrecht, 2009.

3. A. Conca, E. De Negri, A.V. Jayanthan and M.E. Rossi, Graded rings associated with contracted ideals, J. Algebra 284 (2005), 593-626.

4. A. Conca, E. De Negri and M.E. Rossi, Contracted ideals and the Gröbner fan of the rational normal curve, Alg. Numer. Theor. 1 (2007), 239-268.

5. A. Corso, L. Ghezzi, C. Polini and B. Ulrich, Cohen-Macaulayness of special fiber rings, Comm. Alg. 31 (2003), 3713-3734.

6. V. Crispin Quiñonez, Integral closure and related operations on monomial ideals, Ph.D. thesis, Stockholm University, 2006. 
7. V. Crispin Quiñonez, Integral closure and other operations on monomial ideals, J. Comm. Alg. 2 (2010), 359-386.

8. D. Delfino, A. Taylor, W.V. Vasconcelos, R.H. Villarreal and N. Weininger, Monomial ideals and the computation of multiplicities, in Commutative ring theory and applications, Lect. Notes Pure Appl. Math. 231, Dekker, New York, 2003.

9. L.A. Dupont and R.H. Villarreal, Edge ideals of clique clutters of comparability graphs and the normality of monomial ideals, Math. Scand. 106 (2010), $88-98$.

10. C. Escobar, R.H. Villarreal and Y. Yoshino, Torsion freeness and normality of blowup rings of monomial ideals, in Commutative algebra, Lect. Notes Pure Appl. Math. 244, Chapman \& Hall/CRC, Boca Raton, FL, 2006.

11. I. Gitler, C. Valencia and R.H. Villarreal, A note on Rees algebras and the MFMC property, Beitr. Alg. Geom. 48 (2007), 141-150.

12. S. Goto and Y. Shimoda, Rees algebras of Cohen-Macaulay local rings, in Commutative algebra, Lect. Notes Pure Appl. Math. 68, Marcel Dekker, New York, 1982.

13. D. Grayson and M. Stillman, Macaulay2, A software system for research in algebraic geometry, available at http://www.math.uiuc.edu/Macaulay2/.

14. C. Huneke, Complete ideals in two-dimensional regular local rings, in Commutative algebra, Math. Sci. Res. Inst. Publ. 15, New York, Springer, 1989.

15. C. Huneke and I. Swanson, Integral closure of ideals, rings, and modules, Lond. Math. Soc. Lect. Note 336, Cambridge University Press, Cambridge, 2006.

16. C. Huneke and B. Ulrich, Residual intersections, J. reine angew. Math. 390 (1988), 1-20.

17. M. Lejeune-Jalabert, Linear systems with infinitely near base conditions and complete ideals in dimension two, in Singularity theory, World Science Publishing, River Edge, NJ, 1995.

18. J. Lipman, On complete ideals in regular local rings, in Algebraic geometry and commutative algebra, Vol. I, Kinokuniya, Tokyo, 1988.

19. J. Lipman and B. Teissier, Pseudo-rational local rings and a theorem of Briançon-Skoda about integral closures of ideals, Michigan Math. J. 28 (1981), 97-112.

20. S. Morey and B. Ulrich, Rees algebras of ideals with low codimension, Proc. Amer. Math. Soc. 124 (1996), 3653-3661.

21. D. Rees, Hilbert functions and pseudorational local rings of dimension two, J. Lond. Math. Soc. 24 (1981), 467-479.

22. A. Schrijver, Theory of linear and integer programming, John Wiley \& Sons, New York, 1986.

23. Combinatorial optimization, Alg. Comb. 24, Springer-Verlag, Berlin, 2003.

24. A. Simis and W.V. Vasconcelos, The syzygies of the conormal module, Amer. J. Math. 103 (1981), 203-224. 
25. N.V. Trung, Reduction exponent and degree bound for the defining equations of graded rings, Proc. Amer. Math. Soc. 101 (1987), 229-236.

26. W.V. Vasconcelos, Integral closure, Springer Mono. Math., New York, 2005.

27. J. Watanabe, m-full ideals, Nagoya Math. J. 106 (1987), 101-111.

28. O. Zariski, Polynomial ideals defined by infinitely near base points, Amer. J. Math. 60 (1938), 151-204.

29. O. Zariski and P. Samuel, Commutative algebra II, 1960 edition reprint, Grad. Texts Math. 29, Springer-Verlag, New York, 1975.

Departamento de Álgebra, Análisis Matemático, Geometría y Topología AND Instituto de InVestigación en Matemáticas IMUVA, Universidad DE Valladolid, Facultad de Ciencias, 47011 Valladolid, Spain

Email address: pgimenez@agt.uva.es

Departamento de Matemática, Universidade Federal de Pernambuco, 50740560 Recife, PE, Brazil

Email address: aron@dmat.ufpe.br

Department of Mathematics, Rutgers University, 110 Frelinghuysen Rd, PiscataWAy, NJ 08854

Email address: vasconce@math.rutgers.edu

Departamento de Matemáticas, Centro de Investigación y de Estudios Avanzados del IPN, Apartado Postal 14-740, 07000 Mexico City, D.F.

Email address: vila@math.cinvestav.mx 